\title{
EchoGéo
}

$21 \mid 2012$

Pays émergents

\section{Pour une géographie sociale des pays émergents}

Introduction du dossier

Antoine Fleury et Myriam Houssay-Holzschuch

\section{CpenEdition}

Journals

Édition électronique

URL : https://journals.openedition.org/echogeo/13167

DOI : 10.4000/echogeo.13167

ISSN : 1963-1197

Éditeur

Pôle de recherche pour l'organisation et la diffusion de l'information géographique (CNRS UMR 8586)

Référence électronique

Antoine Fleury et Myriam Houssay-Holzschuch, "Pour une géographie sociale des pays émergents », EchoGéo [En ligne], 21 | 2012, mis en ligne le 10 octobre 2012, consulté le 10 août 2021. URL : http:// journals.openedition.org/echogeo/13167; DOI : https://doi.org/10.4000/echogeo.13167

Ce document a été généré automatiquement le 10 août 2021.

EchoGéo est mis à disposition selon les termes de la licence Creative Commons Attribution - Pas d'Utilisation Commerciale - Pas de Modification 4.0 International (CC BY-NC-ND) 


\title{
Pour une géographie sociale des pays émergents
}

\author{
Introduction du dossier
}

Antoine Fleury et Myriam Houssay-Holzschuch

1 Nouvel ordre économique mondial, nouvelles géographies du développement, nouvelles puissances... un éventail d'expressions cherche, depuis bientôt trois décennies, à rendre compte de changements majeurs et multidimensionnels dans l'économie politique du monde globalisé. En particulier, elles décrivent le passage accompli à un monde polycentrique, où la domination occidentale, puis celle de la Triade, sont aujourd'hui contrebalancées par la puissance économique, voire politique, de pays autrefois qualifiés de sous-développés - la Chine en étant la figure archétypique. Ces pays sont aujourd'hui regroupés sous le label de "pays émergents ", rendant ainsi compte de leur arrivée relativement récente dans le concert des puissances. Plus largement, ce trope de l'émergence est fréquemment convoqué pour désigner un ensemble vaste, complexe et mouvant de phénomènes politiques, économiques, sociaux et culturels, aboutissant à des reconfigurations telles qu'on a pu parler, avec le pluriel de rigueur, de « Mondes émergents » (Atlas du Monde diplomatique, 2012). L'émergence est ainsi devenue une nouvelle catégorie, manière de mettre le monde en ordre pour le décrire voire le penser.

2 Cependant, ce label a tendance à osciller entre une ubiquité scientifiquement peu féconde et une focale encore lourdement centrée sur les phénomènes économiques et politiques. Ce dossier ${ }^{1}$ tente d'aller au-delà, à partir d'explorations empiriques, pour montrer en quoi les reconfigurations liées au phénomène d' "émergence » touchent en plein le champ du social, notamment, dans une approche critique, pour y recomposer des relations de pouvoir mais aussi des pratiques. Il s'agit donc de voir comment, dans ce qu'il faut bien appeler le monde majoritaire (majority world) les géographies quotidiennes des acteurs, y compris individuels, se modifient. 


\section{Les enjeux de la catégorisation}

3 En premier lieu, il faut souligner que le terme d'émergence prend sa place dans une histoire des catégories utilisées pour penser le monde, sa diversité, ses disparités et inégalités. On le sait, dire c'est faire, nommer c'est s'approprier : la construction sociale de catégories, spatialisées ou non, est une pratique scientifique commune sur laquelle il faut se pencher de manière critique. Schaffter, Fall et Debarbieux (2010) proposent la triade réification-naturalisation-fétichisation pour cette analyse.

4 Alors qu'on parlait, après la Seconde Guerre mondiale, de « pays en développement ", dans une perspective téléologique, normative et centrée sur le champ économique, puis de «Tiers Monde» - le terme renvoyant à une posture d'affirmation et de revendication politique à l'époque de la Guerre froide - avant d'utiliser l'appellation «Sud», singulier puis pluriel et politiquement plus neutre en apparence car naturalisé (Gervais-Lambony et Landy, 2007), «l'érosion des cadres d'analyse et des modèles politiques ainsi que la grande diversité des résultats économiques ont fait prendre conscience de la multiplicité des trajectoires nationales » (Gabas et Losch, 2008, p. 27), ce qui a donné lieu à l'éclosion progressive de nouveaux termes capables de rendre compte de cette diversité : nouveaux pays industriels (NPI), pays les moins avancés ${ }^{2}$ (PMA), pays en transition (correspondant aux anciens pays du bloc soviétique), etc. L'appellation "pays émergent", aujourd'hui couramment utilisée, s'inscrit dans ce mouvement, même si elle ne renvoie pas à un groupe unifié de pays dans les statistiques internationales non plus qu'à une série de critères quantifiés. Elle est d'abord apparue dans le monde de la finance au cours des années 1980 (Bret, 2011 ; Gabas et Losch, 2008). On commence alors à parler d'économies à marché émergent pour désigner des pays à la croissance rapide et présentant des opportunités d'investissements pour les entreprises des pays riches. Puis elle s'est diffusée par la suite dans les médias et dans le champ académique.

5 Trois grands phénomènes, correspondant chacun à de profondes transformations économiques dans plusieurs groupes de pays, ont contribué à l'affirmation de la catégorie de "pays émergent». C'est en premier lieu les changements dans la géographie de la production industrielle, dans laquelle certains pays «en développement» ont progressivement pris plus d'importance: les "dragons asiatiques » (Hong-Kong, Taiwan, Corée du Sud et Singapour), suivis par les «bébés dragons » (Malaisie, Thaïlande, Indonésie et Philippines) voire les "jaguars» sudaméricains ont attiré l'attention des investisseurs et des financiers, tout comme une série disparate de processus économiques et spatiaux (développement des zones franches, maquiladoras, délocalisations...). Une seconde dimension du processus est apparue avec la mise en place de réformes économiques dans une grande partie de l'ancien bloc communiste, s'engageant dans une transition vers l'économie de marché, avec une phase de croissance importante: politique d'ouverture par les Zones économiques spéciales de la Chine, Doi Moi vietnamien, chute de l'Union soviétique, «européanisation" économique et politique des pays d'Europe centrale et orientale (PECO). Enfin, on observe l'affirmation sur la scène économique et politique mondiale d'un certain nombre de " grands » pays émergents (Chine, Brésil, Russie et Inde) qui se distinguent par la taille de leur territoire et l'importance de leur population, avec son corollaire : un marché intérieur gigantesque. En particulier, la Chine joue ici un rôle majeur: sa croissance économique, ses capacités de production et d'exportation, sa 
puissance financière et créditrice, ainsi que sa place très rapidement devenue majeure dans les géographies mondiales du pouvoir en font un acteur à part, mais aussi archétypique à la fois de l'émergence et du nouveau polycentrisme mondial.

Pour autant, force est de souligner le caractère « remarquablement mouvant » (Sgard, 2008) de la notion d'économie ou de pays émergent, ses « contours en pointillés et des définitions flottantes » (Gabas et Losch, 2008, p. 26) ainsi que « la diversité et la labilité des critères mobilisés pour délimiter de façon rigoureuse cet ensemble " (Piveteau et Rougier, 2010, p. 3). Les institutions internationales, le monde académique et celui de la finance utilisent de fait des critères différents, ce qui explique la très grande variabilité du nombre de pays concernés par cette appellation: la délimitation des "pays émergents " est donc à géométrie variable, ouverte "vers le haut» - avec des pays désormais développés comme la Corée du Sud - aussi bien que "vers le bas » - avec des pays « en voie d'émergence » tels que ceux du Maghreb. On peut en fait identifier deux grandes approches (Gabas et Losch, 2008, p. 30). D'une part, une approche restrictive, centrée sur les " grands pays émergents » que sont Chine, Brésil, Russie, et Inde, définit l'émergence dans une optique d'opposition au G7, avec éventuellement d'autres pays comme l'Afrique du Sud et le Mexique. Cette approche a elle aussi donné lieu à catégorisation avec l'acronyme BRIC, proposé en 2001 par Jim O'Neill, économiste chez Goldman Sachs. D'autre part, une approche plus englobante et plus classique regroupe l'ensemble des pays que l'on appelait jadis " en développement ", à l'exclusion des pays les moins avancés. Autour du noyau dur décrit ci-dessus, cet ensemble agrège donc des pays moins puissants sur les plans économiques et politiques correspondant au second rang des pays émergents (par exemple le Chili et la Turquie), ainsi qu'un troisième cercle constitué de pays fragiles ayant connu des trajectoires heurtées voire descendantes (Argentine, Indonésie) et de pays en voie d'émergence, comme la Thaillande, le Vietnam, la Malaisie, mais aussi l'Egypte ou le Maghreb³.

Ces catégorisations font largement débat et sont l'objet d'instrumentalisations diverses, à l'échelle mondiale mais aussi à l'échelle nationale. Ainsi, la catégorie même de BRIC reste mouvante et objet d'enjeux de pouvoir. En 2010, les pays des BRIC, reprenant à leur compte la terminologie d'O'Neill et se constituant en groupe de pression, ont proposé à l'Afrique du Sud, la principale puissance économique et politique du continent africain, de les rejoindre pour donner naissance aux BRICS. Cette extension de périmètre de l'émergence a été critiquée par O'Neill, ce dernier mettant en avant les faiblesses de la puissance sud-africaine et la dissymétrie entre les quatre géants et le dernier venu. Par le relais de la presse sud-africaine ${ }^{4}$, cette extension donne lieu à débat public sur ce que peut recouvrir le terme d'émergence et son périmètre. En outre, elle va jusqu'à mettre la notion en question : en effet, croissance économique et développement, par exemple indiqué par l'IDH, semblaient jusque là de bons moyens pour cerner le phénomène, mais l'Afrique du Sud, désormais membre de ce qui se rapproche le plus d'une liste officielle des pays "vraiment émergents ", a vu son IDH baisser dans la dernière décennie, du fait des ravages de l'épidémie de VIH/SIDA (Houssay-Holzschuch, 2010). Dès lors, qu'est-ce qu'un pays émergent dont le développement humain recule? Les enjeux d'image, politique comme économique, qu'il y a à " en être » apparaissent aussi a contrario : le même O'Neill a proposé en 2011 les MIST, sorte de session de rattrapage de l'émergence pour le Mexique, l'Indonésie, la Corée du Sud et la Turquie ${ }^{5}$ ! 
8 L'existence de ces enjeux, scientifiques comme politiques, de la catégorisation n'empêche évidemment pas de l'utiliser, bien au contraire. En particulier, les «pays émergents » nous semblent intéressants à analyser non seulement parce que leur existence révèle bien des reconfigurations mondiales, mais aussi parce que la rapidité des changements marquant ces pays permet d'appréhender, en un même lieu, des problématiques tenant traditionnellement des "pays du Nord» (géographie industrielle et financière, formes urbaines comme les communautés fermées ou les gratte-ciel, politiques publiques...), d'autres tenant des «pays du Sud» (pauvreté du plus grand nombre pour reprendre la formule si parlante de Michel Rochefort, importance de l'informel...) et d'autres plus neuves (polarisation sociale, nouveaux mouvements sociaux...). Pour autant, l'utilisation de cette catégorie ne doit pas mener à son essentialisation, laquelle serait paradoxale pour une notion désignant des changements profonds, rapides et multidimensionnels. Elle ne doit pas non plus compartimenter les analyses (ce qui tient aux pays émergents qui s'opposerait à la fois à ce qui tient du « Nord » ou du «Sud) et nuire à une approche ordinariste, comme celle défendue par Jenny Robinson (2006). Plus encore, nous aimerions qu'elle contribue à une géographie décentrée (Robinson 2003), ne construisant plus ses concepts simplement à partir du cœur occidental de la production des savoirs, mais également dans le monde majoritaire, dont font partie les « pays émergents ».

\section{Les approches dominantes de l'émergence en sciences sociales : économie et géopolitique}

9 Par ailleurs, la notion d'émergence telle qu'appliquée depuis les années 1980 à un certain nombre de pays est profondément marquée par des biais disciplinaires. En sciences sociales, ce sont les critères de définition proposés par les économistes qui dominent. Selon C. Jaffrelot (2008), les pays émergents se définissent d'abord par leur "décollage économique », attesté par le poids croissant de certains d'entre eux dans l'économie mondiale (Chine, Inde et Brésil en particulier). Ainsi, le PIB de la Chine a été multiplié par 5,7 entre 1980 et 2008 et celui de l'Inde par 2,2. Les pays émergents se caractérisent également par le fait que ce décollage s'y est produit en étroite relation avec une « insertion accélérée dans l'économie mondiale » (Jaffrelot, 2008), si bien que l'on a pu parler pour ces pays d'une « croissance de globalisation » (Sgard, 2008; Ruet, 2008). L'augmentation de leurs exportations - favorisée par des monnaies sous-évaluées - et leur capacité à attirer les investissements étrangers depuis les années 1990 (Bost, 2004) rendent bien compte du lien entre émergence et globalisation, de même que conjointement "le développement rapide de firmes compétitives et se globalisant " (Piveteau et Rougier, 2010). Plusieurs auteurs soulignent enfin la relative stabilité institutionnelle des pays dits émergents. Ainsi, l'émergence ne peut se comprendre sans être mise en perspective dans un temps plus long, en relation avec des " configurations étatiques spécifiques" (Gabas et Losch, 2008) et avec les politiques publiques poursuivies dans ce cadre (Piveteau et Rougier, 2010). Elle renvoie ainsi à la fois à une insertion dans la globalisation - qui implique l'adoption de nouvelles normes - et à l'affirmation d'États efficaces qui ont su consolider la croissance économique, en se dotant d'instances de régulation des marchés performantes. En définitive, le modèle des pays émergents correspondrait à « une forme d'économie mixte », ces pays ayant su en partie s'émanciper des règles libérales du consensus de Washington ${ }^{6}$ " après que ces 
dernières aient fait leur travail minimal d'institution de l'agent privé, des règles du marché et de sape des régimes corporatistes " (Piveteau et Rougier, 2010, p. 8). Ce modèle se distinguerait de trois autres modèles : celui « des pays qui n'émergent pas du tout ", principalement en Afrique, et dont l'échec serait lié à la faillite de l'État ; celui des " économies rentières ", qui se caractériseraient quant à elles par un hiatus entre d'une part le financement et le gouvernement de l'État et d'autre part l'économie privée et concurrentielle; enfin, celui « des pays qui ont pris le tournant libéral des années 1980, mais sans acquérir ensuite la capacité à conduire des politiques publiques efficaces ", comme le Venezuela, l'Argentine voire la Russie (Sgard, 2008, p. 52-53).

Une autre perspective s'est imposée plus récemment dans les sciences sociales, celle des relations internationales et de la géopolitique. De nombreux auteurs soulignent ainsi l'évolution des puissances qu'implique l'émergence : de puissance économique, le statut des pays émergents se consolide et ceux-ci tendent à s'imposer comme des acteurs importants dans l'actuel système international (Milani, 2011). Jaffrelot (2008) fait même de cette volonté de peser sur les affaires du monde l'un des critères de l'émergence, de même que l'économiste J. Coussy (2008) qui propose un dernier critère pour définir les pays émergents : être en mesure de menacer la position des pays dominants. L'émergence renvoie donc aussi au basculement d'un monde devenant réellement polycentrique, incluant donc la fin de l'hégémonie occidentale. Nombre de ces pays sont d'abord souvent des pôles régionaux, insérés dans des organisations régionales, et déploient, comme par exemple l'Afrique du Sud, une "stratégie d'émergence " (Darbon 2008, p. 142) fondée sur une diplomatie active. Certains se sont associés en 2009 pour former les BRICS (Brésil, Russie, Inde, Chine et Afrique du Sud), club politique visant à fédérer leur action avec une volonté forte de peser diplomatiquement sur l'ordre mondial dont ils s'estiment exclus. Enfin, c'est sans surprise que les pays émergents tendent à développer leur puissance militaire, gage à la fois d'indépendance et de respect des autres puissances, comme c'est tout particulièrement le cas en Inde et en Chine (Giblin, 2011). On retrouve ici le statut ambivalent de la Chine au sein des BRICS: elle est à la fois reconnue en tant que puissance associée par les pays occidentaux et, en même temps, un leader respecté par les pays du Sud; elle a tous les attributs lui permettant de prétendre être, dans les dix ans à venir, la deuxième hyper-puissance (Chambon, 2010). L'émergence se traduit alors entre autres par l'implantation offensive de la Chine et en moindre mesure de l'Inde sur le continent africain, par des pressions diplomatiques sur les pays du Nord (comme en témoigne le lobbying sud-africain pour obtenir un siège permanent de représentant du continent africain au Conseil de Sécurité de l'ONU) ou des investissements croissants dans ces derniers par l'intermédiaire de fonds souverains, ainsi que par de fortes tensions sur les marchés agricoles et énergétiques.

\section{L'émergence, un phénomène multidimensionnel : pour une approche sociale et culturelle}

$11 \mathrm{Au}$ sein des pays émergents, les changements économiques sont accompagnés de changements sociaux et culturels considérables. S'ils ont parfois été désignés par la notion, problématique, de transition (démographique, urbaine, politique, épidémiologique...), il nous semble qu'ils peuvent surtout être compris et analysés comme un "changement social comprimé » parce que particulièrement rapide et de 
grande envergure, si ce n'est systémique. Les sociétés des pays émergents sont traversées par de profondes transformations en lien avec une augmentation globale des niveaux de vie et d'éducation ainsi que par une intensification des circulations - des personnes, biens, informations, idéologies... notamment via les moyens de communication (Appadurai, 1996; Inda et Rosaldo, 2002). Dès lors, l'émergence apparaît comme un phénomène multidimensionnel, qu'il est très réducteur de ne comprendre qu'au travers des champs de l'économie ou des relations internationales alors que les changements sont sociétaux (Bret, 2011; Houssay-Holzschuch, 2007). De plus, ces angles ont tendance à privilégier des approches macro ${ }^{8}$, alors que le niveau méso, mais aussi le niveau micro, sont particulièrement révélateurs des changements et que leur étude permet de mettre en lumière à la fois les «effets locaux de l'émergence ", mais aussi le rôle d'autres acteurs (pouvoirs locaux, acteurs individuels, etc.) pouvant être porteurs d'« un désir local d'émerger » (Morange, 2011, p.348). Alors même que "la notion d'émergence tarde à rejoindre la boîte à outils des géographes " (Morange, 2011, p. 349), en particulier francophones, la géographie sociale et culturelle semble donc bien avoir son mot à dire sur l'émergence.

Dans ce changement majeur qu'est l'émergence, le champ du social est en effet très largement restructuré. Le développement exponentiel de classes moyennes éduquées même si le terme demeure flou - est l'un des faits marquants de cette dernière décennie. Ces classes moyennes tendent à consommer sur le modèle des pays du Nord ou s'en rapprochent, envoient leurs enfants dans les universités, voyagent et sont ouvertes sur le monde extérieur (Bret, 2011). L'automobile et le mall sont les figures archétypales (cf. Erkip, 2003 pour la Turquie) de ces nouveaux modes de consommation, dont la diffusion est soutenue par le développement des moyens de communication (Internet, télévision...). En même temps, cette importance croissante des classes moyennes va de pair avec une polarisation sociale tout aussi croissante : en effet, la recomposition du marché du travail accentue considérablement les disparités en termes d'accès à l'emploi, de revenus comme de conditions de travail. Certes, le processus d'émergence est de nature à créer des emplois, mais le chômage reste une réalité importante dans beaucoup de pays émergents (Bret, 2011), de même que le secteur informel (Roy, 2011; Schneider et al., 2010 ; Simone, 2004 et 2010). Et comme le rappellent Piveteau et Rougier (2010), « les principaux émergents possèdent les indices de Gini les plus élevés révélant une forte concentration des richesses dans le décile supérieur ». C'est l'Amérique latine, en particulier le Brésil, qui est la plus concernée, mais les inégalités se sont également creusées dans la plupart des pays d'Asie au cours des dix dernières années : en Chine, le coefficient de Gini est ainsi passé de 0,3 à la fin des années 1970 à 0,45 en 2002 et selon la Commission chinoise du développement national, les $10 \%$ de la population urbaine les plus riches du pays possèdent $45 \%$ des biens contre 1,4\% seulement pour les $10 \%$ les plus pauvres (Houssay-Holzschuch, 2007). Quant à l'essor de la société de consommation, il alimente les inégalités sociales par les frustrations qu'elle peut provoquer, comme en témoigne la revendication de nouveaux modes de vie et de loisirs (Berry-Chikhaoui et al., 2007).

13 À ces inégalités sociales font écho des inégalités territoriales croissantes, encore une fois liées aux modalités mêmes d'émergence, qui s'effectue le plus souvent de manière partielle et segmentée. L'émergence produit en fait des inégalités à toutes les échelles géographiques (Bret, 2011). Qu'il s'agisse de l'Inde, du Mexique, de la Chine ou du Brésil, les inégalités territoriales sont fortes à l'échelle nationale et se trouvent même 
renforcées par le mécanisme d'émergence. Le cas de la Chine est éloquent : aux régions côtières, qui assurent une grande partie des $10 \%$ de croissance annuelle, s'opposent les régions intérieures et leurs 500 millions de paysans (Sanjuan, 2007). À une échelle plus fine, les grandes villes sont emblématiques de ces inégalités croissantes, comme en témoigne le développement des «Business improvement districts» dans les quartiers centraux (Peyroux et al., 2012) ou de l'enfermement volontaire des plus riches dans des quartiers fermés (Capron, 2006). L'émergence se développe en fait de manière inégale, en lien avec des contextes locaux favorables (développement d'un tissu d'entreprises locales, montée en puissance d'une classe moyenne, présence d'un marché solvable, etc.) et avec la concentration des investissements étrangers dans des localisations particulièrement valorisées pour diverses raisons (coût de l'investissement, législation fiscale, sociale et environnementale, situation plus ou moins favorable pour une insertion dans l'économie mondialisée, etc.) (Bret, 2011). Au final, on a moins affaire à des pays émergents qu'à des territoires émergents - zones d'interface, côtes, «zones franches ", villes voire quartiers urbains - ou à une "émergence locale " (Morange, 2011) qui laisse de côté certains territoires au sein des pays dits émergents. Dans ses modalités spatiales, l'émergence semble davantage correspondre à « une constellation de territoires (...) reliés entre eux par des réseaux d'information et de communication", rappelant "la configuration historique originelle de "l'économiemonde" braudélienne : celle des réseaux de cités " (Gabas et Losch, 2008).

De plus, les disparités économiques entre des pays aux trajectoires différentes ont des conséquences importantes, dans un contexte mondial en pleine recomposition. La globalisation s'accompagne d'un accroissement considérable des échanges et des circulations de tous ordres. Outre l'hétérogénéité désormais bien connue des Nords et des Suds - il y a du Sud dans le Nord et du Nord dans le Sud - les circulations Nord-Sud sont accompagnées de circulations Sud-Nord (flux de personnes à travers les migrations, influences culturelles, etc.) et surtout de flux très importants Sud-Sud (migratoires, économiques, culturels, etc.). Cela entraîne des changements de territorialité par jeu presque systématique sur les échelles (Amin, 2002), des déracinements mais aussi des ré-enracinements avec des spatialités différentes (Inda et Rosaldo, 2002), autant de dynamiques complexes et croisées où les pays émergents apparaissent comme des carrefours majeurs. Ils exportent de la main d'œuvre, à la fois très qualifiée et non qualifiée (Varrel, 2011), souvent dans le cadre de migrations circulaires temporaires, mais accueillent aussi des migrants régionaux. Ils sont également souvent terres de diasporas : terres d'origine (Chine, Inde, Turquie...), terres d'accueil (présence indienne en Afrique du Sud par ex.). Il n'est donc guère étonnant que ces pays soient également dans une posture intermédiaire, à la fois récepteurs et émetteurs, en ce qui concerne la culture. Si Bollywood en est l'exemple archétypal, le cinéma sud-africain est également révélateur : il reçoit les blockbusters américains et envoie en retour à Hollywood Charlize Theron et des films comme Yesterday ou Tsotsi, nommés - et, pour le second, vainqueur - de l'Oscar du meilleur film étranger. Plus encore, il produit son propre blockbuster, District 9, décentrant résolument le regard: pour une fois, les extraterrestres ne se conforment pas à l'hégémonie américaine et n'atterrissent ni à New York, ni à Los Angeles, ni à Washington... mais à Johannesburg. En définitive, de nouvelles formes sociales se mettent en place, faisant jouer de manière dialectique les différentes composantes du changement. Et les pays émergents apparaissent donc comme les plus grands producteurs de ces nouvelles formes sociales et culturelles. 


\section{Quatre entrées principales} leurs dimensions spatiales, pour à la fois renouveler les approches de la géographie sur ces pays et contribuer à améliorer les connaissances sur les pays émergents. Pour rendre compte d'un changement aussi multidimensionnel, de nombreux thèmes étaient possibles: l'affirmation des sociétés civiles et les processus de démocratisation, en particulier via les nouveaux mouvements sociaux; la recomposition des rapports sociaux de sexe; le développement de sociétés de loisirs, de tourisme et plus globalement de consommation ; les évolutions et recomposition du secteur culturel, en termes d'offre comme de pratiques culturelles, ainsi que son rayonnement croissant dans le monde (cinéma par exemple); les politiques urbaines et l'appropriation locale de modèles mondialisés (« mega-projects », question patrimoniale, etc.) ; l'organisation de grands événements (Jeux Olympiques à Pékin en 2008, Coupe du Monde de football en Afrique du Sud en 2010, etc.); les circulations migratoires complexes, combinant migrations et mobilités d'individus très peu qualifiés comme très qualifiés, etc. Toutes n'ont pas été reprises par les auteurs retenus dans ce numéro, et nous regrettons un certain nombre d'absences dans les thématiques ci-dessus, comme de pays émergents non représentés, en particulier en Amérique latine. développe. Marie Gibert et Emmanuelle Peyvel pour le Vietnam, Benjamin Taunay et Philippe Violier pour la Chine s'intéressent aux pratiques socio-spatiales de loisirs et montrent bien comment l'émergence d'une classe moyenne correspond à un « tournant récréatif » faisant rejouer des modèles anciens tout en intégrant de nouvelles façons de faire. Ces pratiques révèlent également les transformations intervenant dans la fabrique des territoires et la construction des identités. Mais le développement de nouvelles pratiques sociales ne va pas sans inégalités. En partant de l'étude de la filière alimentaire à Hanoï, Gwenn Pulliat observe ainsi une bipolarisation croissante entre un groupe dont les pratiques de consommation illustrent l'émergence de la capitale du Vietnam et le groupe des «laissés pour compte» qui ne participent pas à cette évolution.

19 La métropolisation et l'accroissement des mobilités posent la question des transports, en même temps que se repensent les questions de genre. À la croisée des deux 
thématiques, Claire Hancock (pour Mexico et Séoul) et Solène Baffi (pour Cape Town) montrent comment la mobilité des femmes pose des questions spécifiques dans le contexte de villes émergentes, des politiques publiques aux tactiques quotidiennes. On retrouve là encore des inégalités d'accès à la ville qui concernent plus particulièrement des groupes triplement discriminés par leur genre, leur origine sociale et leur race (dans le cas de l'Afrique du Sud), même s'il faut aussi nuancer ces inégalités d'accès à la ville en prenant en compte les «arts de faire» des femmes dans leurs déplacements quotidiens.

D'autres types d'enjeux illustrent la multidimensionnalité de l'émergence. Les deux derniers articles donnent un aperçu des enjeux environnementaux, liés par exemple au développement de la production et à l'extension urbaine (Yves Duchère pour la province d'Hanoi) et, bien évidemment, des changements politiques majeurs qui accompagnent souvent l'émergence, que Delphine Pagès-El Karoui évoque pour l'Egypte.

21 Enfin, la rubrique "Sur l'image » nous entraîne dans les rues et sur les places de Séoul, Mexico, Istanbul, Cape Town et Bangalore, pour analyser les changements spatiaux dans les espaces publics des grandes villes de pays émergents. Ces villes sont le théâtre de tensions spécifiques : hybridation des modèles urbanistiques, arrivée relativement récente de populations d'origine rurale, inégalités sociales et économiques, clivages culturels voire " raciaux », aspirations diverses à la démocratie... autant d'enjeux qui travaillent les espaces publics urbains, dans leurs formes comme dans leurs pratiques.

\section{BIBLIOGRAPHIE}

Amin A., 2002. Spatialities of globalisation. Environment \& Planning A, vol. 34, p. 385-399.

Appadurai A., 1996. Modernity at Large, Cultural Dimensions of Globalization. Minneapolis, University of Minnesota Press.

Berry-Chikhaoui I., Deboulet A., 2001. Les compétences des citadins dans le Monde arabe. Penser, faire et transformer la ville. Paris, Karthala - IRMC - URBAMA.

Bost F., 2004. Les investissements directs étrangers, révélateurs de l'attractivité des territoires à l'échelle mondiale. M@ppemonde, 75, 3, http://mappemonde.mgm.fr/num3/articles/ art04301.html

Bret B., 2011. Un questionnement géographique sur les pays émergents. Bulletin de l'Association de géographes français, vol. 88, n³, p. 227-240.

Capron G. (dir.), 2006. Quand la ville se ferme. Quartiers résidentiels sécurisés. Bréal, collection « D'autre part », $288 \mathrm{p}$.

Chambon J.-L. (dir.), 2010. La Chinamérique : un couple contre-nature? Paris, Eyrolles.

Darbon D., 2008. Afrique du sud : L'utilité comme ressource d'appoint d'une stratégie de puissance émergente, in Jaffrelot C., L'enjeu mondial. Les pays émergents, Paris, Les Presses de Sciences Po. 
Erkip F., 2003. The Shopping Mall as an Emergent Public Space in Turkey. Environment and Planning A, vol. 35, nº, p. 1073-1093.

Gabas J.-J., Losch B., 2008, La fabrique de l'émergence en trompe-l'œil, in Jaffrelot C., L'enjeu mondial, les pays émergents, Paris, Les Presses de Sciences Po, p. 25-40.

Gervais-Lambony P., Landy F., 2007. On dirait le Sud..., Paris, Autrepart, p. 3-14.

Giblin B., 2011. L'Inde et la Chine, pays émergents et puissances mondiales ? Bulletin de l'Association de géographes français, vol. 88, n³, p. 279-295.

Houssay-Holzschuch, M., 2010. Crossing boundaries: vivre ensemble dans l'Afrique du Sud postapartheid. Habilitation à diriger des recherches en géographie, volume 1, Université Paris 1 Panthéon-Sorbonne, http://tel.archives-ouvertes.fr/tel-00542013

Houssay-Holzschuch M. (dir.), 2007. Une géographie des espaces publics dans les pays intermédiaires. Rapport final, ACI Jeunes Chercheurs JC6029.

Inda J. X., Rosaldo R., 2002. The Anthropology of Globalization: a Reader. Oxford, Blackwell.

Jaffrelot C., 2008. Introduction, in Jaffrelot C., L'enjeu mondial. Paris, Presses de Sciences Po, p. 13-22.

Milani Carlos R. S., 2011. Les pays émergents dans l'actuel ordre mondial : changements et légitimité politique. Revue internationale et stratégique, 2/2011, n 82, p. 52-62, www.cairn.info/ revue-internationale-et-strategique-2011-2-page-52.htm

Morange M., 2011. Emergence locale et régénération urbaine au centre-ville au Cap, Bulletin de l'Association de géographes français, vol. 88, n³, p. 348-360.

Peyroux E., Pütz R., Glasze G., 2012. Business Improvement Districts (BIDs): Internationalisation and contextualisation of a 'travelling concept. Guest Editorial, European Urban and Regional Studies, $\mathrm{n}^{\circ} 19$ (2), 111-120.

Piveteau A.,Rougier E., 2010. Émergence, l'économie du développement interpellée. Revue de la régulation, $\mathrm{n}^{\circ} 7$, http://regulation.revues.org/7734

Robinson, J., 2003. Postcolonialising Geography: Tactics and Pitfalls. Singapore Journal of Tropical Geography, 24, p. 273-289.

Robinson, J., 2006. Ordinary cities: between modernity and development. London ; New York, Routledge.

Roy A., 2011. Slumdog Cities: Rethinking Subaltern Urbanism. International Journal of Urban and Regional Research, 35, p. 223-238.

Ruet J., 2008. Penser la figure du capitalisme d'émergence industrielle en Inde et en Chine. Working Paper, Colloque international L'émergence : des trajectoires aux concepts, Bordeaux, 27 et 28 novembre,http://esope.gretha.u-bordeaux4.fr/sites/esope.gretha/IMG/pdf/ penseremergenceruet.pdf

Sanjuan T. (dir.), 2007. Atlas de la Chine. Les mutations accélérées. Paris, Autrement.

Schaffter M., Fall J. J., Debarbieux B. (2009). Unbounded boundary studies and collapsed categories: rethinking spatial objects. Progress in Human Geography, 34, p. 254-262.

Schneider F., Buehn A., Montenegro C. E., 2010. New Estimates for the Shadow Economies all over the World. International Economic Journal, Vol. 24, $\mathrm{n}^{\circ}$ 4, p. 443-461. 
Sgard, J., 2008. Qu'est-ce qu'un pays émergent ?, in Jaffrelot C., L'enjeu mondial, Paris, Presses de Sciences Po, p. 41-54, www.cairn.info/1-enjeu-mondial-1--9782724610871-page-41.htm

Simone A., 2010. City life from Jakarta to Dakar: movements at the crossroads. New York, Routledge. Simone A., 2004. For the city yet to come: changing African life in four cities. Durham, Duke University Press.

Varrel A., 2011. Introduction, in Rajan I., Percot M. (dir.), Dynamics of Indian Emigration. Historical and current Perspectives, Delhi, Routledge.

\section{NOTES}

1. Ce numéro d'Echogéo est en partie le fruit de l'ACI Jeunes chercheurs «Une géographie des espaces publics dans les pays intermédiaires", nJC6029, à laquelle participaient, outre les auteurs de cette introduction, Valérie Gelézeau, Claire Hancock, Bruno Sabatier, Thierry Sanjuan et Annika Teppo. À ce titre, bien des éléments développés ici leur sont redevables. Nos remerciements à eux tous, qu'ils aient pu contribuer à ce numéro ou non. En outre, Myriam Houssay-Holzschuch a bénéficié de l'ANR PERISUD ( $n^{\circ}$ Suds-07-046), dirigée par Jean-Louis Chaléard.

2. Ceux-là définis par une série de critères quantitatifs d'ordre économique.

3. Ainsi, en 2008 , le Boston Consulting Group désigne 14 "économies à croissance rapide » englobant les BRIC plus l'Argentine, le Chili, l'Egypte, la Hongrie, l'Indonésie, la Malaisie, le Mexique, la Pologne, la Thaïlande et la Turquie. La même année, le cabinet PricewaterhouseCoopers évoque 20 marchés émergents, ajoutant à la liste du Boston Consulting Group le Bangladesh, l'Iran, le Nigeria, le Pakistan, les Philippines, l'Arabie saoudite, l'Afrique du Sud et le Vietnam, sans le Chili et la Hongrie.

4. Naidoo, S. (2012). South Africa's presence 'drags down Brics'. Mail \& Guardian, Online, Johannesburg, http://mg.co.za/article/2012-03-23-sa-presence-drags-down-brics

5. The Guardian, 1er février 2011, http://www.guardian.co.uk/global-development/povertymatters/2011/feb/01/emerging-economies-turkey-jim-oneill

6. Le consensus de Washington est un corpus de mesures standard imposées aux économies en difficulté face à leur dette par les institutions financières internationales (Banque mondiale et Fonds monétaire international) et soutenues par le département du Trésor américain.

7. On retrouve dans cette typologie de J. Sgard le problème du périmètre des pays émergents, puisque la Russie, membre des BRICS, en est exclue.

8. Même si certains économistes insistent sur la nécessité de s'intéresser aux stratégies des acteurs aux niveaux national et même local (Gabas, 2008 ; Piveteau et Rougier, 2010 ; Ruet, 2008).

\section{INDEX}

Thèmes : Sur le Champ - Sur le Terrain 


\section{AUTEURS}

\section{ANTOINE FLEURY}

Antoine Fleury, afleury@parisgeo.cnrs.fr , est chercheur CNRS, à l'UMR 8504 Géographie-cités (CNRS, universités Paris 1 Panthéon-Sorbonne et Paris Diderot).

\section{MYRIAM HOUSSAY-HOLZSCHUCH}

Myriam Houssay-Holzschuch, Myriam.Houssay@normalesup.org, est Professeur de géographie à l'Université Joseph Fourier Grenoble 1, PACTE UMR 5194 (CNRS, IEPG, UJF, UPMF) et Institut Universitaire de France. 